\title{
Feedback Searching Bias and Measurement of Its Effect on Ant Colony Optimization
}

\author{
Bolun Chen ${ }^{1,2}$ Ling Chen ${ }^{2,3^{*}}$ \\ ${ }^{1}$ College of Information Science and Technology, Nanjing University of Aeronautics and \\ Astronautics, Nanjing, 210016, China \\ ${ }^{2}$ Institute of Information Science and Technology, Yangzhou University , Yangzhou , China \\ ${ }^{3}$ National Key Lab of Novel Software Tech, Nanjing University, Nanjing, China \\ *yzulchen@gmail.com
}

\begin{abstract}
Keywords: ant colony optimization,deceptive problems,K-cardinality tree problem,solution convergence
\end{abstract}

\begin{abstract}
One of the obstacles in applying ant colony optimization (ACO) to the combinatorial optimization is that the search process is sometimes biased by algorithm features such as the pheromone model and the solution construction process. Due to such searching bias, ant colony optimization cannot converge to the optimal solution for some problems. In this paper, we discover and define a new type of searching bias in ACO named feedback bias. We empirically prove the existence of feedback bias and its influence on ACO. We also present the concept of intensity of bias as a measurement of the effect on ACO by such bias. Our experimental results show that it is reasonable and reliable to use the intensity to measure the influence of bias on ACO.
\end{abstract}

\section{Introduction}

Ant colony optimization (ACO) [1-4] is a popular method for hard discrete optimization problems. Due to its strong ability of optimization, ACO has been used to deal with numerous applications in a large variety of fields[5-16], and demonstrates its excellent performance in combinational optimization.

One of the obstacles in applying ACO to the combinatorial optimization is that the search process is sometimes biased by algorithm features such as the pheromone model and the solution construction process. This behavior caused by the bias is clearly undesirable, because in general it worsens the probability of finding better solutions over time. Blum and Sampels[17,18] studied the application of ACO algorithms to shop scheduling problems. They discovered the bias in the search process. In a similar line of work, Merkle and Middendorf [19,20] studied the bias of a simple ACO by analyzing the dynamics of its model when applied to permutation problems. They discovered that in ACO applied to the permutation problem, the latter decisions of the construction process are entirely biased by the earlier one. Montgomery et al.[21,22] studied the searching bias of ACO on the assignment problem, and attributed search bias to different algorithmic components. They defined two types of searching bias in ACO, namely representational bias and construction bias.

In this paper, we discover and define a new type of searching bias in ACO named feedback bias. We empirically prove the existence and influence of feedback bias in ACO taking the $k$-cardinality tree problem as the test instance. We also present the concept of intensity of bias as a measurement of the effect on ACO by such bias.Our experimental results show that it is reasonable and reliable to use the intensity to measure the influence of bias on ACO. 


\section{Feedback Bias in Ant Colony Optimization}

In this section, we give the definition of the feedback bias. First we define the constrained optimization problem to be solved by ACO.

Definition 1 (Constrained optimization) A constrained optimization problem is defined by a model $P=(S, \Omega, f)$, where $S$ is a search space defined over a finite set of discrete variables, $\Omega$ is a set of constraints on the variables; and $f: \mathrm{S} \rightarrow \mathrm{R}^{+}$is the objective function to be maximized.

In the search space $S$, there are n decision variables $X_{i}, \mathrm{i}=1, \ldots, \mathrm{n}$, where $X_{i}$ can take values from the set $D_{i}=\left\{C_{i}^{1}, C_{i}^{1}, \ldots, C_{i}^{\left|D_{i}\right|}\right\}$. A variable assignment is written as $X_{i}=C_{i}^{j} \quad(i=1,2, \ldots, n)$. A complete assignment to all $X_{i}$ gives a solution instantiation. The set of all such complete assignments is denoted as $S$.

A solution $s \in S$ is called a feasible solution if it satisfies all the constraints in $\Omega$. A feasible solution $s^{*}$ is global optimum if $f\left(s^{*}\right) \geq f(s)$ for all $s \in S$.

Definition 2 (The expected fitness of a solution component) For a solution component $C_{i}^{j}$, we use $G_{i}^{j}$ to denote the set of solutions whose $j$-th component is $C_{i}^{j}$, namely, $G_{i}^{j}=\left\{\left(s_{1}, s_{2}, \ldots, s_{n}\right) \in S \mid s_{i}=c_{i}^{j}\right\}$. We define the expected fitness of solution component $C_{i}^{j}$ as follows:

$$
F\left(c_{i}^{j}\right)=\sum_{s \in G_{i}^{j}} f(s)
$$

Definition 3 (Expected solution) Given a constrained optimization problem $P$, let its optimal solution be $x^{*}=\left(x_{1}^{*}, x_{2}^{*}, \ldots, x_{n}^{*}\right)$. Suppose solution component $C_{i}^{k_{i}}$ has the highest fitness in $c_{i}^{1}, c_{i}^{2}, \ldots, c_{i}^{n}$ :

$$
\arg \max F\left(c_{i}^{j}\right)=c_{i}^{k_{i}} \quad(i=1, \ldots, n)
$$

Solution $c_{\max }=\left(c_{1}^{k_{1}}, c_{2}^{k_{2}}, \ldots, c_{n}^{k_{n}}\right)$ is defined as the expected solution of problem $P$.

Definition 4 (Feedback bias) Given a constrained optimization problem $P$, let its optimal solution be $x^{*}=\left(x_{1}^{*}, x_{2}^{*}, \ldots, x_{n}^{*}\right)$. Let $c_{\max }$ be the expected solution of the problem. ACO algorithm applied to $P$ is said to have a feedback bias if $x^{*} \neq c_{\max }$.

From the definitions, we can see that $c_{\max }$ is the solution consists of the components with the highest expected fitness. Therefore, if feedback bias occurs in ACO, the search process is likely misled to $c_{\max }$, instead of the real optimal solution $x^{*}$. Due to such feedback bias, ant colony optimization cannot converge to the optimal solution for some problems. For instance, when ACO is applied to the $k$-cardinality tree problem, such feedback bias occurs and makes it a second order deceptive system.

\section{The deceptive $\boldsymbol{k}$-cardinality tree problem}

For understanding how the feedback bias affects the performance of ACO for solving deceptive problems, we study this issue on the NP-hard $k$-cardinality tree (KCT) problem, a well-known example of second-order deceptive system for ACO algorithm.

$K$-cardinality tree problem is a generalization of the well-known minimum spanning tree problem . It is defined as follows: Given is an undirected graph $G=(V, E)$, where $|V|=n,|E|=m$, with edge-weights $w(e) \in N^{+}, \forall e \in E$. The set of all trees in $G$ with exactly $k$ edges is henceforth denoted by $\Gamma_{k}$. The goal is to find a solution $S_{k} \in \Gamma_{k}$ that minimizes $W\left(S_{k}\right)=\sum_{e \in S_{k}} w(e)$. We consider the problem of solving 2-cardinality tree as shown in Figure 1. 


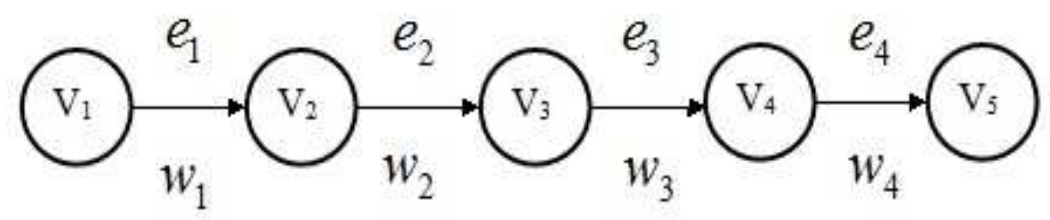

Fig. 1 Instance of 2-cardinality tree problem

The weight settings for this instance are $w_{1}=w_{4}=1, w_{2}=w_{3}=2$. Let the fitness of a solution be the reciprocal of the summation of the weights on the selected edges. The fitness of the solutions are listed in Table 1.

Table 1. Fitness of the solutions

\begin{tabular}{|c|c|c|}
\hline Solution & Summation of the weights & Fitness \\
\hline$S_{0}=\left\{e_{1}, e_{2}\right\}$ & 3 & $1 / 3$ \\
\hline$S_{1}=\left\{e_{2}, e_{3}\right\}$ & 4 & $1 / 4$ \\
\hline$S_{2}=\left\{e_{3}, e_{4}\right\}$ & 3 & $1 / 3$ \\
\hline
\end{tabular}

We denote a solution using a vector $S=\left(s_{1}, s_{2}, s_{3}, s_{4}\right)$. If $s_{i}=1$ then $e_{i}$ is part of the $k$-cardinality tree that is built. It is obvious that the possible solutions are $S_{0}=(1,1,0,0), S_{1}=(0,1,1,0)$, and $S_{2}=(0,0,1,1$,$) . From Table 1$ we can see that $f\left(S_{0}\right)=1 / 3, f\left(S_{1}\right)=1 / 4, f\left(S_{2}\right)=1 / 3$. Obviously, the global optimum is $S_{0}=(1,1,0,0)$ and $S_{2}=(0,0,1,1)$.

When ACO is used to solve the 2-cardinality tree problem, each ant constructs a solution $S=\left(s_{1}, S_{2}, s_{3}, s_{4}\right)$. In each iteration, an ant fixes one bit of $S$. For the $j$-th bit, the ant has two choices $c_{j}^{0}$ and $c_{j}^{1}$, corresponding to setting the $j$-th bit to 0 and 1 respectively.

For the instance in Table 1 , we have $F\left(G_{1}^{0}\right)=1 / 4+1 / 3=7 / 12, F\left(G_{1}^{1}\right)=1 / 3, F\left(G_{2}^{0}\right)=1 / 3$, $F\left(G_{2}^{1}\right)=1 / 4+1 / 3=7 / 12, F\left(G_{3}^{0}\right)=1 / 3, F\left(G_{3}^{1}\right)=1 / 4+1 / 3=7 / 12, F\left(G_{4}^{0}\right)=1 / 4+1 / 3=7 / 12$, $F\left(G_{4}^{1}\right)=1 / 3$. Since $F\left(G_{1}^{0}\right)>F\left(G_{1}^{1}\right), \quad F\left(G_{2}^{1}\right)>F\left(G_{2}^{0}\right), \quad F\left(G_{3}^{1}\right)>F\left(G_{3}^{0}\right), F\left(G_{4}^{0}\right)>F\left(G_{4}^{1}\right)$, the expected solution of the problem is $c_{\max }=(0,1,1,0)$, which is not equal to the optimal solution of the problem $x^{*}=(1,1,0,0)$ or $(0,0,1,1)$. Therefore, there exits feedback bias in ACO for solving 2-cardinality tree problem.

We test the influence of the feedback bias in classical ACO solving the 2-cardinality tree problem. We set the initial value of pheromone $\tau_{j}^{k}$ as $\tau_{j}^{k}(0)=F\left(G_{j}^{k}\right) \quad(k=0,1)$, the probability for an ant to select value $C_{j}^{k}(k=0,1)$ is:

$$
P\left(C_{j}^{k}, t\right)=\frac{\tau_{j}^{k}(t)}{\tau_{j}^{0}(t)+\tau_{j}^{1}(t)}
$$

In each iteration, the pheromone is updated as :

$$
\tau_{j}^{k}(t+1)=\rho \tau_{j}^{k}(t)+\sum_{s \in S_{j}^{k}} f(s) \quad(j=1, \ldots 2 n, k=0,1)
$$

Here, $\rho$ is the evaporation rate and $S_{j}^{k}$ is the set of solutions generated at the $t$-th iteration that have $c_{j}^{k}$ as the $j$-th bit. We make 1000 trials on the instance of 2-cardinality tree problem in Table 1. The experimental results are shown in Table 2. 
Table 2.The experiment results on 2-cardinality tree problem

\begin{tabular}{|c|c|c|c|}
\hline Solution & Number of trials convergence to $S_{\mathrm{i}}$ & Percentage $(\%)$ & Fitness $F\left(S_{i}\right)$ \\
\hline $\mathrm{S}_{0}$ & 33 & 3.3 & $1 / 3$ \\
\hline $\mathrm{S}_{1}$ & 928 & 92.8 & $1 / 4$ \\
\hline $\mathrm{S}_{2}$ & 39 & 3.9 & $1 / 3$ \\
\hline
\end{tabular}

From Table 2 we can see that due to the feedback bias, $92.8 \%$ of the 1000 trials convergence to the expected solution only $7.2 \%$ reach the optimums. This is caused by the feedback bias which directs the ants to move to the expected solution by the pheromone on each solution component.

\section{Intensity of bias on the ACO}

So far in the study of the bias in ACO, there is no a measurement on the effect of bias on the performance of ACO. All the definitions of ACO bias are only a description of its existence which cannot be used to quantifiable evaluate its influence. To make better understanding and analysis of the bias, we give a measurement of the effect of bias on the ACO.

Definition 5 (Intensity of the bias) Given a constrained optimization problem $P$, let its optimal solution be $x^{*}=\left(x_{1}^{*}, x_{2}^{*}, \ldots, x_{n}^{*}\right)$. Let $c_{\max }$ be the expected solution of the problem. The intensity of the searching bias of ACO algorithm applied to problem $P$ is defined as $B(p)=\frac{F\left(x^{*}\right)}{F\left(c_{\max }\right)}$, where $F(x)$ id the fitness of a solution $x$.

We use intensity of the searching bias as a measurement of the effect of bias on ACO. The higher the intensity is, the larger influence of the bias effects the performance of ACO algorithm.

Next we use the 2-cardinality tree problem as an example to show that the measurement we presented is reliable and reasonable to evaluate the effect of the bias on ACO. We fix the fitness of solutions $S_{1}$ at $1 / 4$, and change the fitness of solutions $S_{0}$ and $S_{2}$, denoted as $F(x)$, to obtain different bias intensity $B(p)$. We test the problem and compare the results with different bias intensity so as to verify whether the intensity $B(p)$ is reasonable and reliable to measure the influence of the bias on the performance of ACO.

We set the value of $F(x)$ as $0.05,0.1,0.15,0.2,0.25,0.3$, and perform 1000 trials using each value of $F(x)$. The experimental results are as shown in Table 3.

Table 3.Experimental results with different bias intensity

\begin{tabular}{|c|c|c|c|c|c|}
\hline$F\left(S_{1}\right)$ & $B(p)$ & $\begin{array}{l}\text { Number of trials } \\
\text { with solution } \\
\left\{e_{1}, e_{2}\right\}\end{array}$ & $\begin{array}{l}\text { Number of trials } \\
\text { with solution } \\
\left\{e_{2}, e_{3}\right\}\end{array}$ & $\begin{array}{l}\text { Number of trials } \\
\text { with solution } \\
\left\{e_{3}, e_{4}\right\}\end{array}$ & $\begin{array}{l}\text { Percentage of the trials with } \\
\text { solution }\left\{e_{1}, e_{2}\right\} \text { and }\left\{e_{3}, e_{4}\right\} \\
(\%)\end{array}$ \\
\hline 0.05 & 0.20 & 515 & 6 & 479 & 99.4 \\
\hline 0.1 & 0.40 & 497 & 27 & 476 & 97.3 \\
\hline 0.15 & 0.60 & 397 & 198 & 405 & 81.2 \\
\hline 0.2 & 0.8 & 244 & 506 & 250 & 23.4 \\
\hline 0.25 & 1.00 & 120 & 767 & 113 & 8.7 \\
\hline 0.3 & 1.20 & 42 & 913 & 45 & 23.3 \\
\hline
\end{tabular}

From Table 3 we can see that the percentage of the trials with optimal solution $\left\{e_{1}, e_{2}\right\}$ and $\left\{e_{3}, e_{4}\right\}$ will be increased with the lower $B(p)$ value.This means the $B(p)$ value reflects the influence of the bias on the performance of ACO. Therefore, it is reasonable and reliable to use the intensity as a measurement of the influence of bias on ACO. 


\section{Conclusions}

Due to the searching bias, ant colony optimization cannot converge to the optimal solution for some problems. We discover and define a new type of searching bias in ACO named feedback bias. We empirically prove the existence of feedback bias and its influence on ACO. We also present the concept of intensity of bias as a measurement of the effect on ACO by such bias. Our experimental results show that it is reasonable and reliable to use the intensity to measure the influence of bias on ACO.

\section{Acknowledgments}

This research was supported in part by the Chinese National Natural Science Foundation under grant Nos. 61070047, 61070133 and 61003180, State Key Fundamentals Research(973)Project under contract 2012CB316003, Natural Science Foundation of Jiangsu Province under contracts BK2010318, BK21010134, and Natural Science Foundation of Education Department of Jiangsu Province under contract 09KJB20013.

\section{References}

[1] Dorigo M, Stützle T, Ant Colony Optimization, MIT Press, 2004.

[2] Dorigo M, Blum C, Ant colony optimization theory: A survey, Theoretical Computer Science, 344 (2005) 243-278 .

[3] Blum C, Ant colony optimization: Introduction and recent trends, Physics of Life Reviews, 2 (2005) 353-373.

[4] Shtovba S, Ant Algorithms: Theory and Applications, Programming and Computer Software, 31(2005) 167-178.

[5] Agarwal A., Lim M. H., Er M. J., Chew C. Y., ACO for a new TSP in region coverage, Proceedings of Intelligent Robots and Systems 2005, 2-6 Aug. (2005) 1717 - 1722.

[6] Blum C, Beam-ACO - hybridizing ant colony optimization with beam search: an application to open shop scheduling, Computers \& Operations Research, 32(2005) 1565-1591.

[7] Prokopenko M, Peter Wang, Foreman M, Valencia P, Price D, Poulton G, On connectivity of reconfigurable impact networks in ageless aerospace vehicles, Robotics and Autonomous Systems, 53(2005)36-58.

[8] Kuo R.J., Wang H.S., Hu T. L. , Chou S.H., Application of ant K-means on clustering analysis, Computers \& Mathematics with Applications, 50 (2005) 1709-1724.

[9] R.J. Kuo, Y.P. Kuo, Kai-Ying Chen, Developing a diagnostic system through integration of fuzzy case-based reasoning and fuzzy ant colony system, Expert Systems with Applications, 28(2005)783-797.

[10]Katangur, A.K., Akkaladevi S., Pan, Y., Fraser, M.D., Applying ant colony optimization to routing in optical multistage interconnection networks with limited crosstalk, Proceedings of 18th International Parallel and Distributed Processing Symposium, 2004, pp26-30

[11] Shyong Jian Shyu, B.M.T. Lin , Tsung-Shen Hsiao, Ant colony optimization for the cell assignment problem in PCS networks, Computers \& Operations Research, 33(2006)17131740.

[12] Maniezzo V., Carbonaro A., An ANTS heuristic for the frequency assignment problem, Future Generation Computer Systems, 16(2000)927 - 935. 
[13]Kalinli A., Karaboga N., Artificial immune algorithm for IIR filter design, Engineering Applications of Artificial Intelligence, 18(2005)919-929.

[14]Talbi E.-G., Roux O., Fonlupt C., Robillard D., Parallel Ant Colonies for the quadratic assignment problem, Future Generation Computer Systems, 17(2001)441-449.

[15]Gambardella L. M., Dorigo M.,Ant Colony System hybridized with a new local search for the sequential ordering problem, INFORMS Journal on Computing, 12(2000)237-255.

[16]Lee Z.J., Lee C.Y., Su S.F, An immunity-based ant colony optimization algorithm for solving weapon-target assignment problem, Applied Soft Computing, 2(2002)39-47.

[17]Blum C., Sampels M., Ant colony optimization for FOP shop scheduling: A case study on different pheromone representation, in Proceedings of Congress on Evolutionary Computation 2002, vol. 2, pp. 1558-1563.

[18]Blum C, Sampels M. When model bias is stronger than selection pressure, In Proceedings of the 7th International Conference on Parallel Problem Solving from Nature(PPSN'02), London, UK ,Springer-Verlag, 2002, pp.893-902.

[19]Merkle D, Middendorf M. Modeling the dynamics of ant colony optimization algorithms, Evolutionary Computation, 2002, 10(3) : $235-262$.

[20]Merkle D, Middendorf M. Modeling ACO: composed permutation problems, Proceedings of ANTS2002, Lecture Notes in Computer Science, 2002, 2463:149-162 .

[21]Montgomery J, Randall M, Hendtlass T. Solution bias in ant colony optimization: Lessons for selecting pheromone models, Computers and Operations Research, 2008, 35(9):2728-2749.

[22]Montgomery J, Randall M, Hendtlass T. Structure advantages for ant colony optimization inherent in permutation scheduling problems, Proceedings of IEA/AIE , LNAI, 2005, 3533, :218-228. 